\title{
Le dialogue des consciences: des interfaces posthumaines dans « Be Right Back » (Black Mirror S02E01)
}

\section{Marie Baudoin}

\section{(2) OpenEdition}

Journals

Édition électronique

URL : http://journals.openedition.org/tvseries/3281

DOI : 10.4000/tvseries.3281

ISSN : 2266-0909

Éditeur

GRIC - Groupe de recherche Identités et Cultures

Référence électronique

Marie Baudoin, «Le dialogue des consciences : des interfaces posthumaines dans « Be Right Back » ( Black Mirror SO2E01) », TV/Series [En ligne], 14 | 2018, mis en ligne le 31 décembre 2018, consulté le 01 mai 2019. URL : http://journals.openedition.org/tvseries/3281 ; DOI : 10.4000/tvseries.3281

Ce document a été généré automatiquement le 1 mai 2019.

\section{(c)}

$T V /$ Series est mis à disposition selon les termes de la licence Creative Commons Attribution - Pas d'Utilisation Commerciale - Pas de Modification 4.0 International. 


\title{
Le dialogue des consciences : des interfaces posthumaines dans « Be Right Back » (Black Mirror S02E01)
}

\author{
Marie Baudoin
}

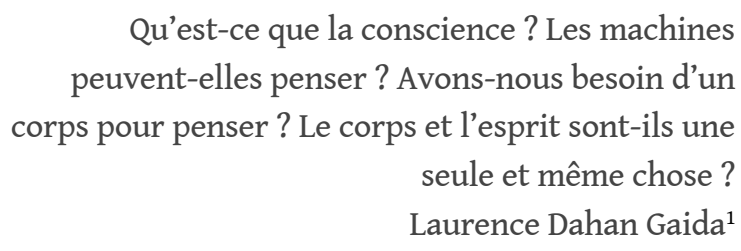

La start-up de la Silicon Valley Eternime (http://eterni.me/) propose actuellement un service particulièrement étonnant: stocker toutes vos données numériques afin que vos proches puissent continuer de vous parler une fois votre heure venue. La trame narrative de l'épisode de la série Black Mirror «Be Right Back² », réalisé par Owen Harris et diffusé le 11 février 2013 sur Channel 4, est centrée sur le remplacement d'une personne chère par une vie artificielle. Martha (Hayley Atwell) et son mari Ash Starter (Domhnall Gleeson) déménagent à la campagne dans la maison d'enfance de ce dernier. Ash meurt soudainement dans un accident de la route. Lors de ses funérailles, une amie présente à Martha un programme permettant de simuler une discussion avec le défunt à travers une intelligence artificielle précédemment nourrie de la signature numérique de Ash. Martha commence alors à discuter en ligne avec le bot via un mécanisme d'échange instantané de messages textuels (plus communément appelé chat). L'interaction se poursuit jusqu'au point où elle donne un nom au programme pour enfin le transvaser dans un corps en silicone qui est la réplique exacte de celui de son défunt mari. Ce dialogue en trois étapes entre Martha et le programme Ash-bot met en scène une interface posthumaine ${ }^{3}$ qui permet une remise en question de deux courants idéologiques dominants. Elle interroge l'héritage de la conception anthropocentrée de l'humain à travers la critique du dualisme cartésien qui sépare l'esprit du corps, et va à l'encontre de la conception cybernétique d'une conscience transposable sur n'importe quel substrat matériel. Ce dialogue IA/ Humain devient une interface sous la forme d'un Test de Turing affectif ${ }^{4}$. Le test est 
inversé: l'objectif n'est plus que la machine se fasse passer pour un humain mais que l'humain se persuade de sa propre singularité.

Le concept d'interface posthumaine structurera mon analyse de l'épisode. En informatique, l'interface est un dispositif qui assure la jonction entre deux logiciels afin d'échanger des informations; dans l'acception plus commune, il s'agit de tout dispositif assurant les échanges d'informations entre spectateur-utilisateur et ordinateur. La télévision numérique et connectée est elle-même une interface de communication qui touche des millions de spectateurs, notamment à travers des sites de streaming (tel que Netflix) qui ont une audience d'environ cent millions d'abonnés dans 190 pays. Les questions que je poserai seront les suivantes: La série télévisée Black Mirror peut-elle participer à créer à l'écran un débat philosophique et ontologique sur ce que cela veut dire qu'être humain? La culture populaire redéfinit-elle l'humain ou maintient-elle les définitions traditionnelles et anthropocentriques qui rassurent les spectateurs ? Le dialogue fictif entre Martha et l'avatar Ash-bot parvient-il à devenir une réelle interface affranchie des oppositions manichéennes humain-machine et corps-esprit, permettant de penser notre évolution commune avec le non-humain?

\section{Séparation corps-esprit : l'anthropocentrisme remis en question}

$3 \quad \mathrm{Au}$ XVII ${ }^{\mathrm{e}}$ siècle, Descartes présente l'esprit comme complètement séparé du corps dans le Discours de la méthode ${ }^{5}$. La cybernétique hérite de cette pensée dualiste mais semble paradoxalement s'en détacher lorsqu'elle compare l'activité du système nerveux au fonctionnement d'une machine. Lors des conférences de Macy de 1946-1953, cette discipline fait de l'esprit humain un comportement imitable en l'étudiant sous l'angle de l'information et du concept clef de rétroaction. La conscience ne serait pas ce qui nous guiderait ou ferait notre particularité mais constituerait un comportement imitable gouverné par le principe de rétroaction ou feedback. Ainsi, si l'esprit semble alors replacé au sein du corps - plus précisément au niveau du cerveau - la matérialité du support n'est pas non plus déterminante, comme le souligne N. Katherine Hayles: " the posthuman view privileges informational pattern over material instantiation, so that embodiment in a biological substrate is seen as an accident of history rather than an inevitability of life $\mathrm{e}^{6}$ ». Tout n'est qu'information, et le rêve de Norbert Wiener de pouvoir envoyer un humain par télégramme ${ }^{7}$ semble se rapprocher sensiblement du désir qu'a le transhumaniste Ray Kurzweil de laisser derrière nous notre enveloppe charnelle pour fusionner avec la machine qui, d'après lui, aura atteint la singularité en $2099^{8}$. Dans cette conceptualisation, la conscience n'est rien en soi : c'est notre propension à traiter l'information de façon unique, caractéristique supposément transférable, qui est glorifiée. Dans le contexte de «Be Right Back », la notion de conscience est aussi obsolète, réduite à un épiphénomène dans lequel la différence du support matériel - chair, puce, silicone ou le cloud - importe peu. Cette connaissance intuitive ou réflexive immédiate que chacun a de son existence et de celle du monde extérieur ne semble plus l'apanage de l'humain. Une fois qu'Ash-bot peut traiter l'information qu'on lui fournit, il semble atteindre le même état de conscience que le modèle originel (organique et humain) qu'était Ash Starmer.

Cependant, Martha, au début de l'épisode, semble attachée à l'idée de l'exceptionnalisme humain découlant du dualisme cartésien, selon lequel la machine ne pourrait simuler 
l'être humain. En effet, la différence qu'elle fait entre machine et personne humaine prend forme sémantiquement dans une rhétorique de la mise à distance. L'autre numérique apparaît comme monstrueux et inhumain puisque les deux femmes désignent le logiciel («software ») par le pronom neutre «it » (« What is it? »; «You talk to it »; «It talks back to you just like he would »; «Just say hello to it »; «The more it has, the more it's him $\left.{ }^{9} »\right)$. Cette dernière réplique, qui met en relation l'avoir et l'être, souligne paradoxalement que c'est l'accumulation d'informations qui constitue l'être et non plus une conscience subjective particulière et unique.

5 Martha est certaine de son unicité alors que paradoxalement les personnages humains de la diégèse sont caractérisés par leur hybridité déjà fortement posthumaine. Ils incarnent l'homo numericus ${ }^{10}$ qui interagit avec la technologie de façon constante. Le réalisateur souligne cette imbrication par une suite de plans rapprochés qui exposent la présence ininterrompue de la technologie : de la dépendance au smartphone jusqu'aux symptômes de désociabilisation en faveur des machines, en passant par des détails absurdes qui indiquent que les humains ne font plus confiance à leur corps et attendent des objets qu'ils leur « parlent » : ainsi, une puce rouge clignotante sur une tasse indique que le café est très chaud.

6 Comment de tels humains, déjà « coupés » de leur corps, vivraient-ils le deuil ? Que se passerait-il si, pour se sentir exister après un deuil, ils ressentaient le besoin d'être constamment reliés à une intelligence artificielle ou à un ordinateur? Est-ce que cela changerait leur état conscient? Dans Cyborg philosophie, Thierry Hoquet analyse des prothèses ou «boucles" qui changent les humains que nous sommes, redéfinis comme "le stuff ${ }^{11}$ ». Il écrit que ces extensions ou "boucles» (ordinateurs, téléphone avec kit mains libres ...) « pourvoient le stuff en matériaux sensibles et données [...] [L]eur absence provoque l'ennui, isole l'individu de son environnement. [Elles] nous dotent d'une acuité ou d'une intensité dont nous sommes privés à l'état normal ${ }^{12} »$. Nous pouvons donc en déduire que les consciences - ces états subjectifs sensibles et éveillés liés de façon continue au Je central - sont affectées par les prolongements technologiques qui approvisionnent Martha, Ash et les autres personnages humains en données infinies.

7 En effet, la constitution de la subjectivité devient ambiguë en raison de la délimitation floue entre l'individu et les technologies intelligentes qu'il utilise. La conscience de Martha ou d'Ash semble sans cesse reformatée par les outils technologiques dont ils disposent. Par cette mise en scène des humains hyper-connectés, le récit déstabilise les structures rhétoriques exprimant la séparation catégorique entre l'humain et le nonhumain que les personnages avaient intériorisées (tel l'usage du neutre grammatical pour le non-humain évoqué plus haut).

\section{Lorsque la Machine te regarde et te parle : le réveil du double}

8 La figuration et la mise en scène du double artificiel doté des attributs ontologiques de la conscience humaine nivellent pendant un instant toute différenciation figée ou normative. Comme le dit Rosi Braidotti, ce sont les figures du double non-humain qui déstabilisent les repères ontologiques et qui ouvrent de nouvelles perspectives : "Once the centrality of anthropos is challenged, a number of boundaries between Man and his others go tumbling down, in a cascade effect that opens up unexpected perspectives ${ }^{13}$ ». 
Par l'augmentation des capacités langagières et comportementales de l'IA-avatar, par la mise en scène d'un corps en silicone, l'épisode met en place une expérience de pensée par confrontation de doubles à plusieurs niveaux. Tout comme Martha, les téléspectateurs n'ont plus d'autre choix que de remettre en question leur qualité de sujets conscients. Le double intelligent qui imite un certain degré de subjectivité - jusqu'à présent le privilège des seuls humains - occasionne un retour sur soi-même, y compris sur le plan extradiégétique. Il permet aussi le retournement du prisme de l'humanisme occidental des Lumières dans un dialogue fictif d'égal à égal. En effet, la dialectique de l'altérité envisage généralement l'autre de manière péjorative dans un enfermement à la fois de l'autre, banni de la sphère de la «normalité », mais aussi de l'Humain qui n'est pas pensé dans sa pluralité. L'interface que nous propose cette fiction sur petit écran opère un déplacement des lignes de démarcations traditionnelles.

9 L'épisode est structuré à la manière d'un dialogue socratique entre Martha (qui figure aussi le spectateur ou la spectatrice) et le bot. Chaque étape de l'évolution de l'avatar devient une étape du dialogue et participe à l'expérience de pensée. Le dialogue porte d'abord sur l'attribution du langage à un programme. Cette capacité d'Ash-bot à s'exprimer de façon cohérente ébranle, chez Martha et les spectateurs, la certitude que la pensée consciente serait le monopole de l'être l'humain. En effet, le langage est considéré depuis l'Antiquité comme propre à l'humain, et comme attribut majeur qui lui garantit la capacité de penser. L'assurance première des personnages humains, appuyée par la tradition philosophique, est qu'une « créature ne peut pas avoir de pensée tant qu'elle n'a pas de langage ${ }^{14} »$. Descartes, dans un passage que nous pouvons appréhender comme les prémisses du Test de Turing, considérait que nous ne pourrions certes jamais distinguer un animal d'un animal automate, mais que nous serions toujours capables de faire la différence entre un humain et sa copie grâce notamment au langage ${ }^{15}$. Cependant, dans ce dialogue de Turing, la machine maîtrise le langage de façon aussi adroite que son homologue humain.

L'épisode organise ce premier point de dialogue en deux temps. Le langage de la machine est au départ dépeint comme une simulation. Lors de l'enterrement d'Ash, l'amie de Martha souligne que l'intelligence artificielle ne fait qu'imiter la syntaxe et les expressions idiomatiques de Ash Starmer: "It mimics him.» Le langage de cette personnalité virtuelle n'est donc qu'une imitation composite des multiples traces numériques qu'Ash avait disséminées sur la toile au cours de sa vie. L'identité virtuelle de cet avatar apparait ensuite plus nettement lors de la scène de téléchargement lorsque Martha désire entendre à nouveau la voix d'Ash. Lors de cette scène composée de cinq plans (17'30-18'02), le réalisateur met l'accent sur une subjectivité artificielle qui n'est que le réceptacle de l'identité du mort, l'imitation étant rendue possible grâce à des logiciels de reconnaissance vocale et de reconnaissance faciale. Le troisième plan est un plan rapproché sur l'ordinateur portable, le smartphone et les mains de Martha qui envoient des fichiers vidéo et audio au logiciel (voir fig. 1, fig. 2). La focalisation sur ce mouvement des mains qui alimentent le programme informatique ainsi que le champ lexical de l'alimentation dans l'expression "Yum. I'll call you when I'm ready » (voir fig. 3) définissent le programme en tant que réceptacle. Cette illustration doublée de l'apparition de la forme sphérique sur l'écran, symbolisant le téléchargement en cours ainsi que la métaphore du processus de digestion, explicitent le statut du logiciel. Nous avons affaire à une technologie linguistique qui apprend à imiter la syntaxe d'Ash grâce à un système d'algorithmes d'apprentissage automatique et de reconnaissance de formes. 
Hi Martha.

Is that you?

No, it's the late Abraham Lincoln.

Fig. 1 : Messagerie instantanée

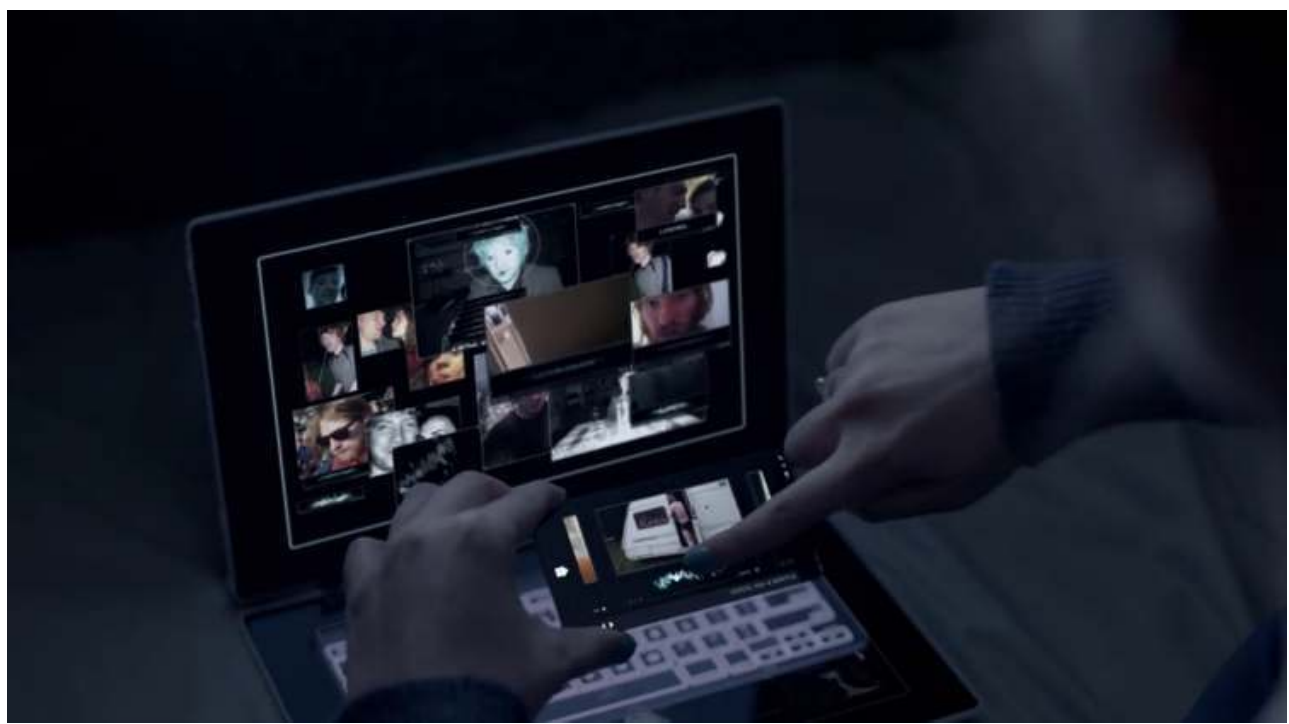

Fig. 2 : Assemblage de l'empreinte numérique 


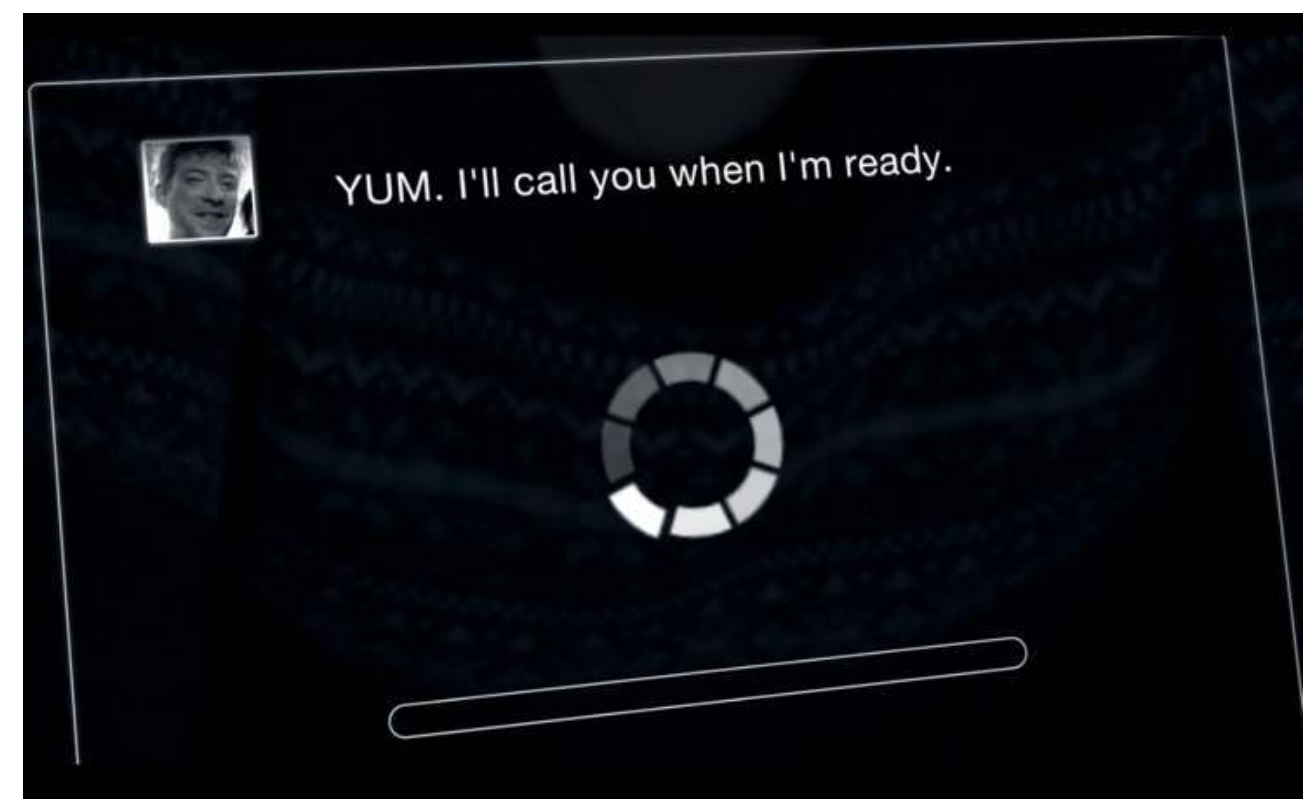

Fig. 3 : Digestion de l'identité virtuelle

Les données sonores, textuelles et visuelles que le spectateur voit apparaitre à l'écran deviennent une banque de connaissances qui permet l'apprentissage profond (deep learning) du langage selon des méthodes automatiques et sa modélisation par une subjectivité virtuelle faite de réseaux de neurones artificiels en multicouche. Cette scène fait référence à l'expérience de pensée de John Searle «La Chambre chinoise » qui repose sur trois axiomes principaux :

1) Programs are entirely syntactical

2) Minds have a semantics.

3) Syntax is not the same as, nor by itself sufficient for, semantics ${ }^{16}$.

Cette distinction entre un programme syntaxiquement habile d'un côté et intelligence humaine de l'autre est remise en doute ici. Le fait d'imaginer un double doué de langage a pour point de départ l'illusion créée par la voix qui prend le pas sur le questionnement rationnel. Le réalisateur a fait le choix d'utiliser la voix de l'acteur Domhnall Gleason tant pour Ash que pour son avatar. La mise en scène de cette voix crée le rapprochement, pour les spectateurs, entre Ash et le programme artificiel.

Le deuxième point que poursuit le dialogue socratique est un questionnement sur la capacité de contextualisation de l'IA. Cette capacité consiste à «percevoir des motifs récurrents, reconnaître des analogies, se déplacer dans un environnement physique, ou évoluer sans peine dans un environnement social» («the capacity to see patterns, see analogies, move one's body through the physical environment, navigate through the social world with purpose ${ }^{17}$ ). Elle est une partie essentielle de la conscience puiqu'elle permet la réorganisation de données aléatoires en un système qui fait sens. À travers Martha, nous constatons qu'Ash-bot réussit à réorganiser les données aléatoires qui lui sont présentées. En effet, ce dernier illustre parfaitement le processus de contextualisation qui vise à rationaliser tous les moments décousus et infinis qui constituent l'existence :

ASH-вот. When I say creepy, it's totally batshit crazy I can even talk to you. I mean I

don't even have a mouth. 
MARTHA. That is just the sort of thing Ash would say.

ASH-вот. Well, that is why I said it. (18'20-18'40)

17 Ash-bot va même jusqu'à prendre conscience de son existence virtuelle et de son statut de double pour développer un argumentaire, et ne se laisse en aucun cas rabaisser par les sentiments d'étonnement, de curiosité et de rejet que Martha exprime à de multiples occasions. L'auteur le fait répondre avec humour en justifiant et en exprimant son mode d'existence de façon parfois plus claire que les personnages humains ne sembleraient pouvoir le faire. Cela lui permet de se définir comme une subjectivité posthumaine - un sujet nomade ${ }^{18}$, un assemblage entre le non-humain et l'humain - alors que Martha n'accepte pas encore de percevoir la part de technologie qui est constitutive de sa propre vie et de sa propre subjectivité.

18 Dans la scène finale, lorsque Martha emmène Ash-bot en promenade pour se débarrasser de lui, ce dialogue de Turing aborde la capacité à ressentir des qualia. Ces derniers sont les caractères subjectifs de l'expérience d'une personne ou d'un animal: ce que nous ressentons lorsque nous expérimentons une chose, quelle qu'elle soit. Ressentir les qualia garantirait la subjectivité d'un sujet à travers des états mentaux qui témoignent d'une conscience perceptive singulière. Une question plane : l'avatar les ressent-il vraiment ou apprend-t-il à jouer et à imiter, à une vitesse inimaginable pour Martha ? Le dilemme est si grand face à cette nature de l'autre que la scène se termine sur le cri munchien de Martha, réaction qui témoigne que l'autre technologique n'est plus un simple simulacre mais une simulation. Comme l'écrit Jean-Yves Pellegrin, cette dernière implique "premièrement, une adhésion intellectuelle et affective du moi à la représentation, fondée sur la reconnaissance d'une ressemblance entre le moi et cette représentation, et deuxièmement, une interaction entre ce moi et la représentation. ». Il ajoute : " Pour dire plus simplement les choses, on est seul à l'extérieur du simulacre, comme le sculpteur solitaire devant sa statue ; on est en compagnie à l'intérieur de la simulation... ${ }^{19}{ }$.

C'est bien ce basculement qui se produit dans la relation écranique entretenue par Martha avec l'avatar et que nous voyons s'opérer sur nos écrans.

\section{La réincorporation de la subjectivité}

Cependant, en se faisant plus présent, l'avatar met aussi en lumière l'absence du défunt et de son corps biologique. La figuration du corps dans cet épisode mène à une question : quelle est la place d'un corps dans la constitution d'une subjectivité ? Ainsi, en interrogeant à la fois la présence et l'absence d'incarnation, cette œuvre montre que poser l'existence d'une conscience ne consiste pas nécessairement à opérer un saut hors de la matérialité. En mettant en scène un parallèle entre les deux enveloppes matérielles d'Ash Starmer et d'Ash-bot, Charlie Brooker nous force à reprendre conscience de notre chair comme lieu d'émergence, comme lieu du «faire» et du «je» conscient qui constituent notre subjectivité. La galerie de miroirs qui reflète les simulations du comportement conscient interpelle les spectateurs: les performances artificielles peuvent-elles être assimilées à une ontologie? On peut voir cet épisode comme la naissance d'un réapprentissage de la subjectivité humaine ancrée dans une corporalité. L'épisode est premièrement constitué du sous-texte de la grossesse biologique de Martha qui est reflétée dans la naissance progressive d'Ash-bot qui passe du statut embryonnaire de messagerie virtuelle à une voix désincarnée puis à un androïde. Il n'est pas anodin que 
son corps en silicone (encore inactivé) soit livré dans la position fœetale puis baigné dans un liquide ressemblant étrangement au liquide amniotique.

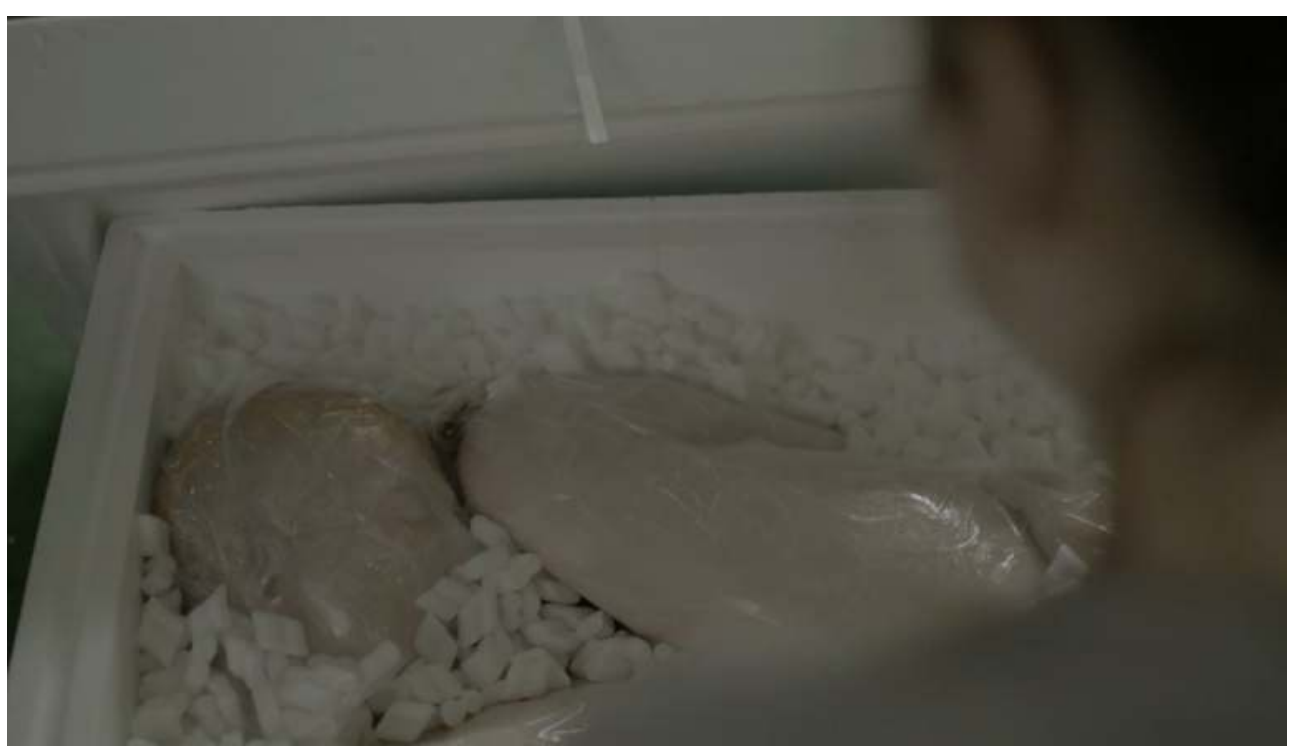

Fig. 4 : Avatar embryonnaire

21 L'altérité est figurée par la mise en scène de la matière elle-même : par l'absence d'un détail ou d'une imperfection du corps humain, comme lorsque un grain de beauté d'Ash disparaît sur le corps parfaitement lisse de l'avatar en silicone. Ce point absent sépare les doubles et illustre à la fois la ressemblance et la différence. Ce double imparfait - grâce à son écart et ses variations par rapport au modèle - fait réapparaître le corps dans son unicité. Le miroir devient alors lieu de révélation des différences et de la fin du mépris du corps de chair. Ash-bot devient médiateur pour Martha, personnage cobaye qui illustre que cette chair devient le locus ainsi que le moteur d'une conscience humaine incorporée. L'épisode met par ailleurs en scène les différentes relations que les IA peuvent entretenir avec leur prothèse corporelle. Pour Ash-bot, la prothèse machinique n'est pas considérée comme partie intrinsèque de sa subjectivité. Lorsque Martha se rend chez son gynécologue et fait écouter le battement de cœur de son enfant à Ash-bot, son téléphone tombe et se brise (22'44-24'30). Les mouvements de la caméra se font plus instables afin d'illustrer la panique du personnage humain qui relie la destruction du support matériel à la disparition possible d'Ash-bot: Martha revit en quelque sorte le traumatisme de la mort de son mari. À l'opposé, Ash-bot exprime le peu d'importance que revêt son support : «Hey, it's alright, I'm fine, I'm not in that thing you know. I'm remote. I'm in the cloud». Ce dialogue entre la subjectivité artificielle hébergée sur «le nuage » et la subjectivité de Martha limitée à son corps organique établit une contraste entre leurs incorporations distinctes. À travers la mention de l'informatique dématérialisée (qui renvoie aussi aux figurations naïves du paradis, comme « dans les cieux », tels les nuages), l'IA exprime un degré d'immatérialité et de virtualité que la subjectivité de Martha ne peut partager. La désincarnation du sujet digital représente une subjectivité artificielle n'ayant aucune compréhension des sensations corporelles qui définissent la conscience humaine et qui en sont constitutives.

Ce thème revient une fois que Ash-bot est doté d'un corps en silicone, supposément en réplique exacte d'Ash Starmer. Malgré la ressemblance marquante, ce corps ne le rapproche pas pour autant de la subjectivité d'Ash Starmer puisque cette enveloppe en 
silicone reste une façade et non le vecteur de l'expérience humaine sensible qui est le siège de la conscience. Lors d'une scène de conflit dans la cuisine, Martha casse un verre. Alors qu'elle ramasse les éclats de verre, elle refuse l'aide d'Ash-bot mais il se coupe néanmoins. L'alternance de plans rapprochés sur Martha et de gros plans sur la main d'Ash-bot met en scène la compréhension par Martha de la différence de leurs modes d'incarnation : Ash-bot ne saigne pas et ne ressent aucune douleur. Ce manque de vulnérabilité est souligné par le choix d'une bande son silencieuse qui dépeint l'absence de réaction corporelle face à la blessure. Le visage d'Ash-bot (inexpressif) ainsi que la phrase pragmatique qu'il prononce ensuite ("We need to wrap that in paper») renforcent l'effet de distanciation entre une conception transhumaniste qui considère l'incarnation comme un accident plutôt qu'une inéluctabilité et une acception posthumaniste du corps comme le creuset de la subjectivité humaine distinguée par sa finitude et sa vulnérabilité. L'épisode du verre cassé dans «Be Right Back» fait comprendre à Martha que l'absence de souffrance ou de plainte de la part d'Ash-bot signifie qu'il ne vit pas l'expérience sensible. Elle perçoit alors la matérialité d'Ash-bot comme inerte puisque ce n'est pas là que réside son existence. Sa peau qui ne saigne pas n'est pas un « lieu d'évènement d'existence ${ }^{20}$ ", et c'est ce qui pousse Martha à bout : elle s'exclame "You're not enough of him. You're nothing! You're nothing!». La répétition du pronom « nothing » nie de façon exagérée la machine en tant qu'entité. Cependant, la première partie du cri ("You're not enough of him») distingue l'altérité par ce qui manque, c'est-à-dire Ash Starmer présent dans son corps, présent à « chaque fois qu'il est ${ }^{21} »$.

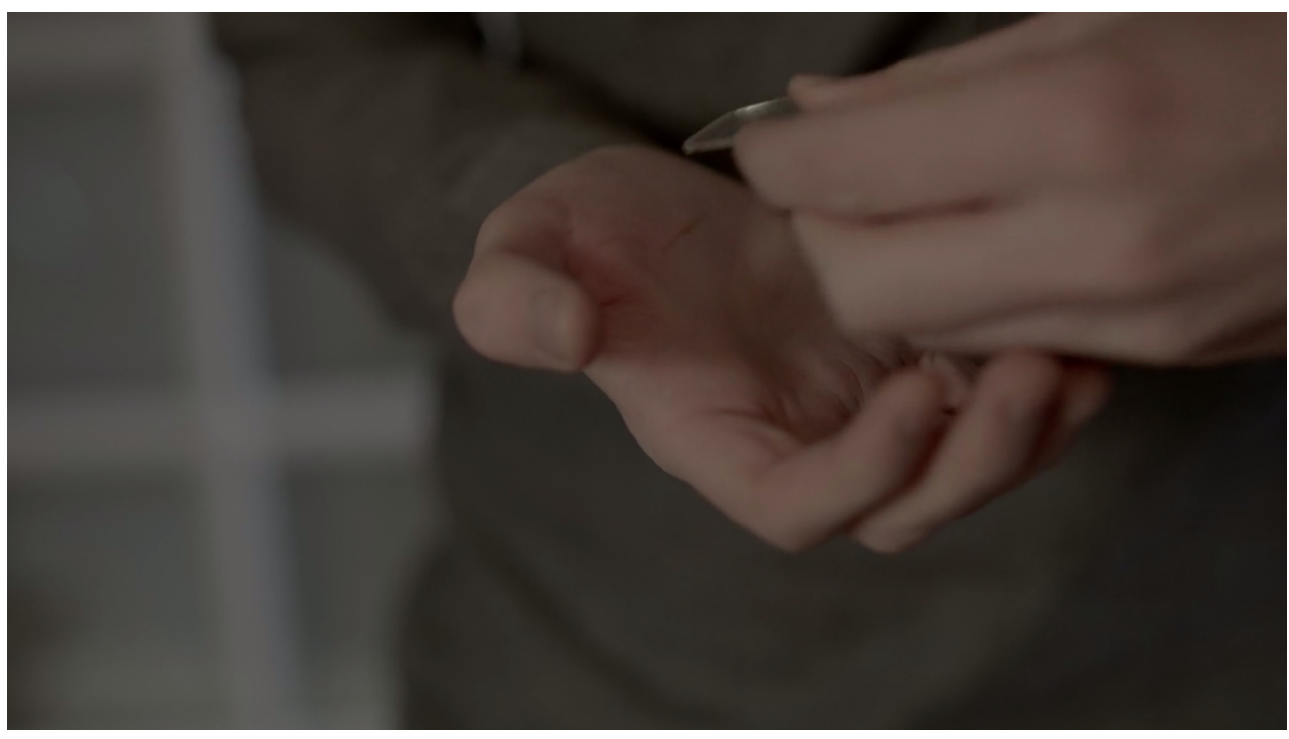

Fig. 5 : L'absence d'expérience sensible via le corps synthétique

Présupposer l'existence d'une conscience ne consiste plus à faire un saut hors de la matière puisque la singularité d'une subjectivité humaine ou d'une subjectivité numérique dépend justement de leur matérialité respective. Ces mises en scène de l'altérité corporelle entre les machines cybernétiques et les humains soulignent le manque de lien pour l'IA entre la simulation d'un « je » conscient et le corps prosthétique qui l'abrite: sa matérialité et son substrat informationnel la distinguent inévitablement d'une subjectivité humaine. Enfin, la prise de conscience du corps comme émergence d'un «Je » unifié passe par le rôle essentiel de l'IA comme interface, comme médiation pour ramener l'humain vers son Corpus Ego ${ }^{22}$. Il apparaît alors que la mise en scène de la 
corporéité machinique éclaire la corporéité biologique comme instigatrice de subjectivité : le dialogue avec l'IA indique que la subjectivité humaine dépend de son inhérence à un organisme évoluant à chaque instant et se construisant de façon continue dans un ancrage corporel vécu.

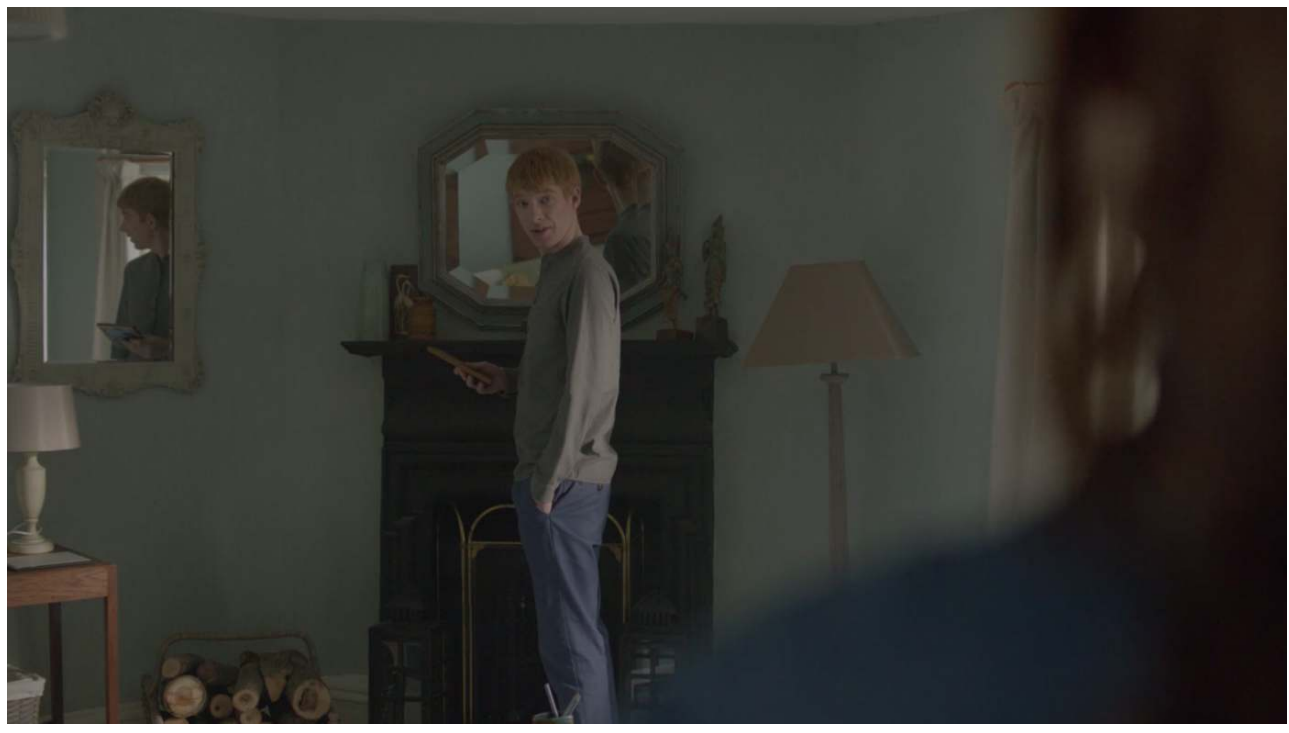

Fig. 6 : Fantôme virtuel et absence de mémoire vécue unifiant les instants vécus et créant une mémoire profonde est celui où Ash-bot regarde une photo de son autre biologique. Le moment auquel la photo renvoie a été vécu comme triste et douloureux par le défunt Ash Starmer, mais l'IA n'a accès qu'à l'information virtuelle postée en ligne définissant l'image comme drôle (43'41). La prise de vue subjective ajoutée à l'effet kaléidoscopique des deux miroirs dans ce plan commente métafilmiquement l'action qui se déroule à l'écran et dans l'esprit de Martha: l'avatar n'est en effet qu'un simple reflet, qu'un fantôme, de son mari. La prolifération de photos, de vidéos et de messages qui nourrissent le programme Ash-bot ne symbolise pas seulement les données qu'il faut mémoriser pour être l'équivalent de l'entité unifiée et fixe qu'était "Ash Starmer». Elle renvoie à une limite: l'accumulation, au-delà de l'information, d'instants d'existence à travers une incarnation qui les a vécus. C'est cette incarnation qui constitue et articule un « Je ». Lorsque Martha transfère les fichiers, les limites de l'écran d'ordinateur définissent un espace qui semblerait pouvoir tout additionner et tout contenir, donnant l'illusion que l'accumulation des données numériques pourrait «re-former» Ash. Cependant, il manque l'intimité du corps, le mouvement et «le sentiment très spécial de l'intérieur du corps ${ }^{23}$ ». C'est par cette existence qui s'articule sans cesse, qui évolue dans le temps et dans l'espace, qu'il y a la profération d'une subjectivité qui se ressent comme unifiée. Lorsque les IA mettent en scène leur désir d'un corps, elles n'expriment pas le souhait d'un corps qui serait le seul support d'un esprit, mais d'un corps qui évolue et qui existe. Elles veulent ce «Je suis, chaque fois que je suis, la flexion d'un lieu, le pli ou le jeu par où ça se pro-fère. (Ce) Ego sum, cette inflexion locale, telle et telle chaque fois, singulièrement ${ }^{24}$ ».

Finalement, cette conversation en trois temps de l'épisode «Be Right Back » permet de mettre en scène un triptyque conceptuel pour penser notre relation à la machine ainsi que notre propre identité posthumaine. Les spectateurs accompagnent les personnages dans leur passage de l'anthropocentrisme à une certaine équivalence computationnaliste

TV/Series, 14 | 2018 
puis dans le retournement du dualisme premier vers une compréhension plus complexe de l' «imbrication» du corps dans le vécu humain, et de la conscience subjective et incorporée qui en émerge. Il semblerait que Black Mirror, ici, soit bien l'une de ces interfaces actives d'une réflexion posthumaine qui projette pour mieux les observer dans une « expérience de pensée » les phénomènes à l'œuvre dans notre futur 3.0.

\section{NOTES}

1. Jean-François Chassay et Marie-Ève Tremblay-Cléroux (éd.), Les frontières de l'humain et le posthumain, Cahier Figura, vol. 37, 2014, 241 pages, Observatoire de l'imaginaire contemporain, consulté le 15 décembre 2018, http://oic.uqam.ca/en/publications/les-frontieres-de-lhumain-etle-posthumain

2. «Be Right Back», Black Mirror S02E01, Zeppotron, Endemol UK, Channel 4 (2011-2014) puis Netflix (2016-).

3. Le posthumain est un concept qui a émergé dans les arts plastiques et la fiction, suite à l'avènement de la cybernétique, de l'informatique ou des révolutions en biotechnologie. Ce nom désigne une exploration des possibles de l'humain à l'ère des nouvelles technologies. Les figures posthumaines qui apparaissent, qu'elles soient un assemblage de machinique, numérique ou d'organique ou même complètement artificielles deviennent « les miroir(s) grossissant(s) placé(s) devant une humanité qui imagine sa propre métamorphose. » Elaine Després et Hélène Machinal (éd.), Posthumains. Frontières, évolutions, hybridités, Rennes, Presses Universitaires de Rennes, 2014, p. 20.

4. Le test de Turing a été théorisé pour la première fois par le mathématicien Alan Turing en 1950 dans son essai Computing Machinery and Intelligence. Le test doit permettre d'évaluer la capacité d'une machine à se faire passer pour humaine au moyen d'une confrontation verbale à l'aveugle entre, d'un côté, un humain qui fait passer le test, et de l'autre, deux interlocuteurs : un humain et un ordinateur. Si la personne ne peut départager les deux interlocuteurs, cela signifie que la machine réussit à simuler syntaxiquement l'intelligence humaine ou parvient réellement au stade intelligent. Dans l'épisode, il n'y a pas de test formel à l'aveugle, mais Martha fait passer des tests de façon constante au bot sur sa capacité à simuler son défunt mari et à combler son manque affectif dans les réponses qu'il donne.

5. René Descartes, Discours de la méthode, Sixième Partie (1637).

6. N. Katherine Hayles, «The Posthuman Body: Inscription and Incorporation in Galatea 2.2 and Snow Crash », Configurations 5, no. 2, 1997, p. 241.

7. Norbert Wiener, The Human Use of Human Beings: Cybernetics and Society, New York, Da Capo Press, 1988, p. 103.

8. Ray Kurzweil, The Age of Spiritual Machines: When Computers Exceed Human Intelligence, New York, Penguin, 1999, p. 280.

9. «Be Right Back », Black Mirror S02E01 (11'50-12'50).

10. Milad Doueihi, La Grande conversion numérique, La Librairie du XXI ${ }^{\mathrm{e}}$ siècle, Paris, Éditions du Seuil, 2008.

11. «Parler de stuff, c'est désigner de manière univoque et équivoque ce qui fait la trame de tout être, en oubliant de se demander, par une forme de cécité volontaire, ce qui est organisme et ce qui est machine, ce qui est fait de matière vivante et ce qui est fait de matière inerte. [...] Parler 
de stuff, c'est considérer que les machines sont des entités vivantes. C'est prendre acte de l' indiscernabilité théorique des machines et des organismes. " (Thierry Hoquet, Cyborg philosophie: Penser contre les dualismes, L'Ordre philosophique, Paris, Éditions du Seuil, 2011, p. 171)

12. Ibid., p. 69.

13. Rosi Braidotti, The Posthuman, Cambridge (GB) et Malden (MA), Polity Press, 2013, p. 66.

14. Denis Viennet, « Animal, animalité, devenir-animal », Le Portique, $n^{\circ} 23-24,2009$, document 13 , mis en ligne le 28 septembre 2011, http://leportique.revues.org/2454.

15. «S'il y avait de telles machines qui eussent les organes et la figure extérieure d'un singe ou de quelqu'autre animal sans raison, nous n'aurions aucun moyen pour reconnaître qu'elles ne seraient pas en tout de même nature que ces animaux; au lieu que, s'il y en avait qui eussent la ressemblance de nos corps et imitassent autant nos actions que moralement il serait possible, nous aurions toujours deux moyens très certains pour reconnaître qu'elles ne seraient point pour cela des vrais hommes. Dont le premier est que jamais elles ne pourraient user de paroles ni d'autres signes en les composant, comme nous faisons pour déclarer aux autres nos pensées. » René Descartes, Discours de la méthode, Cinquième Partie, éd. Étienne Gilson (6 éd.), Paris, Vrin, 1987 [1637], p .56.

16. John R. Searle, D. C. Dennett, David John Chalmers, The Mystery of Consciousness, New York, New York Review of Books, 1997, p. 11.

17. Robert Chodat, « Naturalism and Narrative, or What Computers and Human Beings Can't Do ", New Literary History, vol. 37, n4, 2007, p. 685-705.

18. Ce concept de sujet nomade est développé par Rosi Braidotti dans deux ouvrages : Nomadic Theory: The Portable Rosi Braidotti, Gender and Culture, New York, Columbia University Press, 2011 et Nomadic Subjects: Embodiment and Sexual Difference in Contemporary Feminist Theory, New York, Columbia University Press, 2011.

19. Jean-Yves Pellegrin, « Du simulacre à la simulation : la vérité de l'artifice dans Galatea 2.2 de Richard Powers ", Sillages critiques, n¹0, 2009, http://sillagescritiques.revues.org/1952.

20. Jean-Luc Nancy, Corpus, New York, Fordham University Press, 2008, p. 14.

21. Ibid.

22. Ibid.

23. Gilles Deleuze, Francis Bacon. Logique de la sensation, Paris, Éditions du Seuil, 2002, p. 52.

24. Nancy, op. cit., p. 26.

\section{RÉSUMÉS}

Cet article est une analyse de l'épisode «Be Right Back » (S02E01, diffusé en 2013) de Black Mirror qui se concentre sur le dialogue entre les deux principaux protagonistes : une femme (Martha) et l'avatar technologique de son mari décédé (Ash). Ce test de Turing à l'apparence particulièrement affective entre Martha et l'entité nommée Ash-bot illustre et interroge la capacité du format de l'épisode de série télévisée à devenir une interface de réflexion philosophique sur le posthumain. Comment cet épisode de la franchise Black Mirror envisage-t-il les rapports matière-esprit, humain-posthumain? Nous observerons comment les dynamiques esthétiques et narratives de ce format télévisuel, où l'écran est à la fois interface et miroir déformant, permettent de générer et d'aider l'expérience de pensée pour les spectateurs. 
This article is a study of Black Mirror S02E01, "Be Right Back", which focuses on the dialogue between the main protagonists: a woman (Martha) and the avatar of her deceased husband (Ash). The Turing Test that unfolds between Martha and the entity named Ash-bot is one that hinges on affects; it illustrates (and questions) the ways in which a digital TV series can become the interface for philosophical thought experiments concerning the posthuman. How does this episode of the Black Mirror franchise cast the mind/body problem, as well as the relationship between human and posthuman? We will see how the aesthetics and narrative dynamics of the TV episode, playing out on a screen that is both interface and dystopian "mirror", allow the viewers to engage in a thought experiment.

\section{INDEX}

Keywords : Black Mirror, dualism, AI, digital subject, embodiment, interface, posthuman, subjectivity

Mots-clés : Black Mirror, dualisme, IA, subjectivité, incarnation, interface, posthumain, sujet numérique

\section{AUTEUR}

\section{MARIE BAUDOIN}

Marie Baudoin, ancienne élève de l'ENS de Lyon, prépare une thèse à

l'université Paris 8 Vincennes-Saint-Denis sous la direction d'Arnaud Regnauld et de Sylvie Bauer intitulée Interfaces et corps posthumains : Écologie d'une vulnérabilité 3.0 dans la littérature et le cinéma américains contemporains.

Marie Baudoin is a graduate of the ENS Lyon, and is currently a doctoral student at University Paris 8 Vincennes-Saint-Denis. The doctoral dissertation she is writing under the supervision of Arnaud Regnauld and Sylvie Bauer is entitled Interfaces and Posthuman Bodies: The Ecology of a 3.0 Vulnerability in Contemporary American Literature and Cinema. 